REVIEW

\title{
New approaches for evaluation of soil health, sensitivity and resistance to degradation
}

\author{
Yakov KUZYAKOV (ه) ${ }^{1,2}$, Anna GUNINA ${ }^{3}$, Kazem ZAMANIAN ${ }^{1}$, Jing TIAN ${ }^{4}$, Yu LUO ${ }^{5}$, Xingliang XU ${ }^{6,7}$, \\ Anna YUDINA ${ }^{8}$, Humberto APONTE ${ }^{9}$, Hattan ALHARBI ${ }^{2}$, Lilit OVSEPYAN ${ }^{10}$, Irina KURGANOVA ${ }^{10}$, \\ Tida GE ${ }^{11}$, Thomas GUILLAUME ${ }^{12}$
}

1 Department of Soil Science of Temperate Ecosystems, Department of Agricultural Soil Science, University of Goettingen, 37077 Göttingen, Germany

2 College of Food and Agriculture Sciences, King Saud University, Riyadh 11451, Saudi Arabia

3 Department of Environmental Chemistry, University of Kassel, 37213 Witzenhausen, Germany

4 College of Resources and Environmental Sciences, National Academy of Agriculture Green Development, Key Laboratory of Plant-Soil Interactions (Ministry of Education), China Agricultural University, Beijing 100193, China

5 Institute of Soil and Water Resources and Environmental Science, Zhejiang Provincial Key Laboratory of Agricultural Resources and Environment, Zhejiang University, Hangzhou 310058, China

6 Institute of Geographic Sciences and Natural Resources Research, Chinese Academy of Sciences, Beijing 100101, China

7 CAS Center for Excellence in Tibetan Plateau Earth Sciences, Chinese Academy of Sciences (CAS), Beijing 100101, China

8 Department of Soil Physics and Hydrology, V.V. Dokuchaev Soil Science Institute, Moscow 119017, Russia

9 Center for Research in Mycorrhiza and Agri-Environmental Sustainability (CIMYSA), University of La Frontera, Temuco, Chile

10 Institute of Physicochemical and Biological Problems in Soil Science, Russian Academy of Sciences (RAS), Moscow 142290, Russia

11 State Key Laboratory for Managing Biotic and Chemical Threats to the Quality and Safety of Agro-products, Key Laboratory of Biotechnology in Plant Protection of Ministry of Agriculture of China and Zhejiang Province, Institute of Plant Virology,

Ningbo University, Ningbo 315211, China

12 Laboratory of Biogeosciences, Institute of Earth Surface Dynamics, University of Lausanne, CH-1015 Lausanne, Switzerland

\begin{abstract}
Assessment of soil health requires complex evaluation of properties and functions responsible for a broad range of ecosystem services. Numerous soil quality indices (SQI) have been suggested for the evaluation of specific groups of soil functions, but comparison of various SQI is impossible because they are based on a combination of specific soil properties. To avoid this problem, we suggest an SQI-area approach based on the comparison of the areas on a radar diagram of a combination of chemical, biological and physical properties. The new approach is independent of the SQI principle and allows rapid and simple comparison of parameter groups and soils. Another approach analyzing the resistance and sensitivity of properties to degradation is suggested for a detailed evaluation of soil health. The resistance and sensitivity of soil properties are determined through comparison with the decrease of soil organic carbon (SOC) as a universal parameter responsible for many functions. The SQI-area and resistance/sensitivity approaches were tested based on the recovery of Phaeozems and Chernozems chronose-
\end{abstract}

Received December 21, 2019; accepted March 27, 2020

Correspondence: kuzyakov@gwdg.de quences after the abandonment of agricultural soils. Both the SQI-area and the resistance/sensitivity approaches are useful for basic and applied research, and for decisionmakers to evaluate land-use practices and measure the degree of soil degradation.

Keywords ecosystem stability, land quality, land degradation, soil health, soil management, soil organic matter

\section{Introduction}

Soils are a fundamental part of the biosphere and contribute essentially to cycles of all biophilic elements, ecosystem productivity and stability, and are thus the cradle of life on our planet. Soil health, also referred to as soil quality ${ }^{[1]}$, is therefore commonly defined very broadly as "the capacity of a soil to function within ecosystem and land-use boundaries to sustain biological productivity, maintain environmental quality, and promote plant and animal health" ${ }^{2,3]}$. This definition reflects many linkages between soil functions and soil-based ecosystem services. As these soil functions are crucial for ecosystem services, scientific and practical tools are necessary to evaluate soil 
health. Human activities and climate change alter the environment and their cumulative impacts will determine the future health of biomes and soils ${ }^{[4,5]}$.

Soils have an extremely broad range of functions related to climate, hydrology, biodiversity, food security, land restoration and human health ${ }^{[1,6,7]}$. The functions of natural soils are commonly much more diverse than those of agricultural ones because humans optimize only one function, namely crop production ${ }^{[8]}$ by converting natural ecosystems to agroecosystems ${ }^{[9]}$. Disregarding other functions (e.g., biodiversity, cycles of water and elements, decomposition and sorption of pollutants) while focusing on only one unavoidably leads to soil degradation $^{[9]}$. Therefore, most agricultural soils are degraded compared to their natural ancestors ${ }^{[10]}$. Thus, we define soil degradation as a reduction of functions or intensity of functionality as compared to functions under natural conditions $^{[9]}$. The main questions of this paper are how to assess soil degradation especially considering multifunctionality, and so, what are the approaches to evaluate soil health and its contribution to the stability of (agro) ecosystems?

The evaluation of soil fertility is comparatively simple in contrast to soil health and is based on the productivity of crops. However, this soil fertility view is also focused on only one function, crop productivity, and disregards the whole range of other functions: the multifunctionality. Various approaches for evaluation of soil degradation, quality and health have been suggested to overcome this limitation ${ }^{[1]}$. Most of these approaches are based on the combinations of few chemical, physical and biological soil properties with or without weighting factors and will be termed soil quality index (SQI). In addition to the (partly subjective) selection of the soil properties considered in such a SQI, two main disadvantages limit their applicability: (1) the comparison of various SQI between studies is very difficult, and partly impossible because diverse soil parameters and weighting factors are applied, and (2) the sensitivity of individual parameters to soil degradation depends on the degradation direction (chemical, physical and biological), specific degradation factors (e.g., excessive fertilization, high mechanical loading and chemical pollution) and degradation types (e.g., compaction, aggregate destruction, acidification and erosion). Individual chemical, physical and biological properties therefore become various weights in the final SQI scores.

Here, we suggest, explain and apply two approaches to overcome these SQI disadvantages. The first approach, the SQI-area approach, is based on the comparison of the area on a radar diagram which is produced by all soil parameters contributing to the SQI. The second approach, the resistance-sensitivity approach, reflects the sensitivity of the individual soil parameters to degradation as related to the decrease of organic carbon content.

\section{Calculating SQI-area to compare approaches}

The SQI-area approach is based on a comparison of the area inside of the radar chart produced from the group of individual soil parameters contributing with any weighting (in most cases without weighting or linear weighting ${ }^{[11,12]}$ ) to the SQI (Fig. 1). Each individual soil parameter should be standardized to unity (1.0) for non-degraded soil (in most cases a natural ancestor or analog soil $)^{[10,13,14]}$ (Eq. (1)).

$$
s t P_{i}=\frac{P_{\text {deg }}}{P_{\text {nat }}}
$$

where $s t P_{i}$ is standardized parameter $i, P_{d e g}$ and $P_{n a t}$ are the values of the parameter in the degraded and natural (nondegraded) soils, respectively. Such standardization ensures the comparison of any SQI consisting from any number of parameters. Based on the standardized parameters, the radar diagram can be built for both the non-degraded (natural) soil (all values are maximum, Fig. 1) and the degraded soil. The comparison (e.g., the ratio) of the area covered by both soils reflects the overall intensity of degradation. The corresponding area $\left(\operatorname{Area}_{S Q I}\right)$ will be equal to the sum of individual triangles comprising the whole figure:

$$
\text { Area }_{S Q I}=0.5 \cdot \sum_{i}^{n} s t P_{i}^{2} \cdot \sin \left(\frac{2 \cdot \pi}{n}\right)
$$

where $n$ is the number of parameters used for the SQI, and $\pi(3.14)$.

Despite the total area depending slightly on the number of parameters involved in the SQI calculation, the ratio of areas between degraded and non-degraded soils is independent. The ratio between the sum of all triangle areas in the degraded soil to the area covered by all parameters for natural or undisturbed soil will reflect the overall degradation intensity (Fig. 1).

Due to most of soil parameters decreasing with degradation, it is simple to calculate their standardized values based on undisturbed soil and then to compare the relative decrease in the parameters in the degraded or intensively used soil (the principle: more is better ${ }^{[15]}$ ). For those parameters increasing with soil degradation (e.g., bulk density), the opposite should be used, i.e., minimum value should be assigned to undisturbed natural soil (less is better), and the increased value will be compared. For the parameters, which have an optimal value for most of the soil functions (e.g., $\mathrm{pH}$, water and air permeabilities, hydrophobicity), the difference from the optimum should be used.

The advantages of this area-based (SQI-area) approach are: 


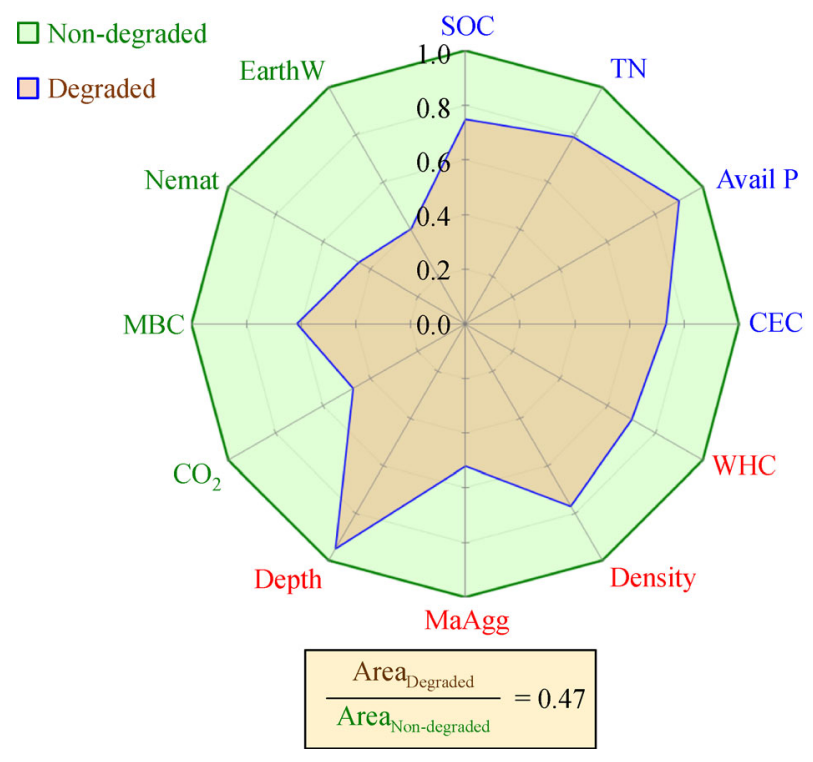

Fig. 1 The soil quality index area (SQI-area) approach suitable for unifying evaluation of any number of soil parameters and to compare various SQI methods. A decrease in the area on the radar plot between a non-degraded and a degraded soil is necessary. The ratio between the SQI area of non-degraded and degraded soils is independent of the number of parameters and the weightings involved in the calculation of the area. Chemical soil parameters are indicated in blue, physical in red, and biological in green. SOC, soil organic carbon content; TN, total nitrogen content; Avail P, available phosphorus; CEC, cation exchange capacity; WHC, water holding capacity; Density, soil bulk density; MaAgg, macroaggregates; Depth, depth of $\mathrm{Ah} / \mathrm{Ap}+\mathrm{B}$ horizons; $\mathrm{CO}_{2}$, $\mathrm{CO}_{2}$ efflux from soil; $\mathrm{MBC}$, microbial biomass carbon content; Nemat, nematodes; and EarthW, earthworms. The ratio of the areas of degraded to non-degraded SQI in this example is 0.47 , reflecting that on average half of the properties and functions are lost by degradation. Note that the values on the degraded soil plot are arbitrary (not experimental); for examples of experimental data see the references ${ }^{[10,13]}$.

- Independence of the results (degradation evaluation) from the number of soil parameters involved in the SQI calculation. Despite individual areas being slightly dependent on the parameter numbers the ratio between areas of degraded and non-degraded soils is independent. Various SQI (calculated by different principles and considering various number of parameters) can therefore be easily compared between studies.

- Comparison with non-degraded soil is required (see the disadvantages given below). This gives a clear overview of how strong the summary of all functions decreased, and thus, the strength of the overall degradation.

- The SQI-area approach provides simple comparison of the efficiency of various soil types or land-use practices.

- The approach simplifies the evaluation of the relative sensitivity and resistance of individual soil parameters to degradation and informs about which soil properties we should be aware of first during new management practices.
Thus, according to the theoretical example in Fig. 1, macroaggregates, $\mathrm{CO}_{2}$, nematodes and earthworms are especially sensitive (maximum decrease; commonly biological properties, see below) to soil degradation. In contrast, available phosphorus and soil depth are resistant (minimum decrease; commonly the physical properties) to degradation.

- It is simple to compare the decrease between the groups of soil properties (i.e., chemical, physical and biological; indicated in various colors in Fig. 1).

- It is irrelevant in which units the parameters are measured (such as $\mathrm{g} \cdot \mathrm{kg}^{-1}$ and $\mathrm{Mg} \cdot \mathrm{ha}^{-1}$ ) because all parameters are standardized with the same parameter in undisturbed soil.

- If the thresholds of complete degradation are known, it is easy to recognize how close the soil is to complete or irreversible degradation. This allows the definition of degradation classes, the thresholds between them, and noreturn points after which the soils cannot be recovered (irreversible degradation).

- Effects of restoration practices and soil recovery on individual properties can be easily evaluated (see below). Thus, the soil progradation, improvement of the properties (reverse of degradation), can be assessed.

- Finally, the SQI-area approach provides a useful visualization and estimation of the decrease in individual parameters.

There is lack of clarity with the application of the SQIarea approach in the case of soil progradation: if the SQIarea for native soil is less than that for agriculturally used (e.g., Fig. 2(b) and Fig. 3, Chernozem 29 years). If the SQIarea of the agriculturally used soil is larger than 1.0, then its functionality is larger or broader than that of the native analog.

The key disadvantage of this SQI-area approach is that the non-degraded or natural soil is necessary for standardization. This limitation, however, is the same for all other SQI approaches ${ }^{[1]}$. Another minor limitation is the necessity to prepare such SQI calculation and visualization of each soil depth or horizon, if soil quality should be estimated not only for the topsoil.

In contrast to some other SQI produced based on principal component, redundancy or discriminant analysis $^{[16]}$, the SQI-area approach does not allow the assessment of the multicollinearity of individual parameters. As with most other SQI approaches, the cultivation time is not considered by the SQI-area approach, and thus, the intensity and duration of individual degradation processes cannot be evaluated. Given that soil properties react to degradation at specific rates (see Kuzyakov and Zamanian $^{[9]}$, de Bruyn and Abbey ${ }^{[17]}$ ) the sensitivity of individual parameters to degradation should be considered.

To present experimental application of the SQI-area approach we selected the data from two soil restoration chronosequences during long-term abandonment of degraded agricultural soils under deciduous forest (Luvic 

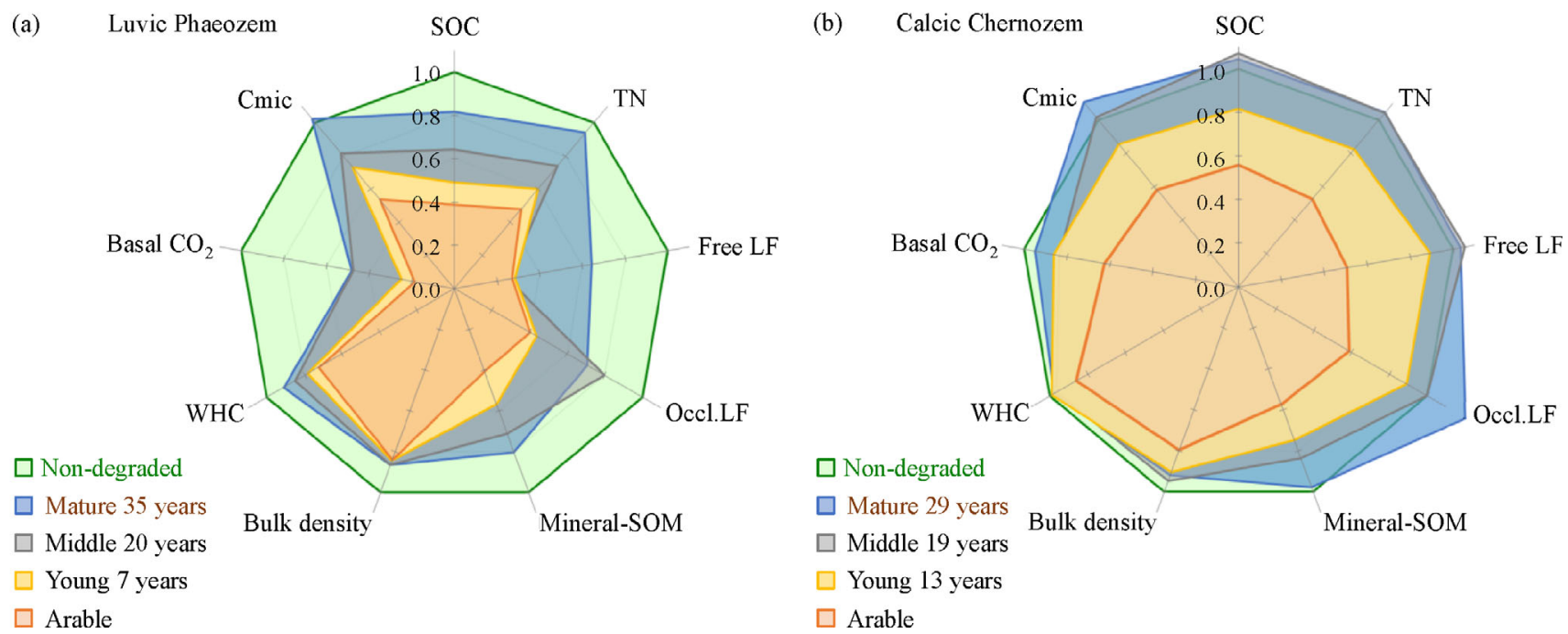

Fig. 2 The Soil Quality Index area (SQI-area) approach presented on examples of two chronosequences of recovery of agricultural soils after abandonment and natural succession under deciduous forest: Luvic Phaeozem (a); and under dry steppe: Calcic Chernozem (b) (data from Ovsepyan et al. $\left.{ }^{[10]}\right)$. The arable, young, middle, mature and non-degraded soils represent the recovery stage. The number close to the recovery stage shows the years of abandonment reflecting the recovery period. The measured soil properties are: SOC, soil organic carbon; $\mathrm{TN}$, total nitrogen; Free LF, free light fraction $\left(\rho<1.6 \mathrm{~g} \cdot \mathrm{cm}^{-3}\right)$; Occl.LF, occluded light fraction $\left(\rho<2.0 \mathrm{~g} \cdot \mathrm{cm}^{-3}\right)$; Mineral-SOM, heavy fraction $\left(\rho>2.0 \mathrm{~g} \cdot \mathrm{cm}^{-3}\right)$; WHC, water holding capacity; Basal $\mathrm{CO}_{2}$, basal respiration; Cmic, microbial biomass carbon.

Phaeozem) and under dry steppe (Calcic Chernozem ${ }^{[10]}$ (Fig. 2). The data clearly show much stronger degradation of the Phaeozem than the Chernozem under agricultural use over several hundred years. The SQI-area of the degraded Phaeozem ( $>150$ years under agricultural use) increased from 0.22 to 1.0 of the natural Phaeozem over 70 years (Fig. 2). In contrast, the SQI-area of the degraded Chernozem ( $>200$ years under agricultural use) retained its functions at $39 \%(\mathrm{SQI}-\mathrm{area}=0.39)$ and had already

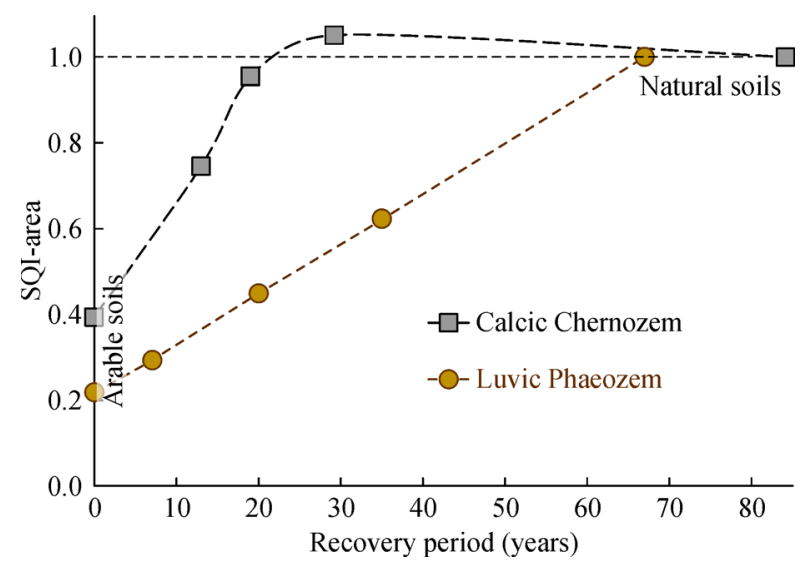

Fig. 3 Development of Soil Quality Index area (SQI-area) during recovery of agricultural soils after abandonment and natural succession under deciduous forest: Luvic Phaeozem; and under dry steppe: Calcic Chernozem ${ }^{[10]}$. The area and the parameters are presented in Fig. 2. recovered after 20 years (Fig. 3). These radar diagrams indirectly reflect the most sensitive (basal respiration, free light fraction in Phaeozems) and resistant (bulk density, water holding capacity) parameters according to their decrease compared to non-degraded soils (Fig. 2).

\section{Sensitivity and resistance of soil parameters to degradation}

Sensitivity and resistance of soil parameters to degradation reflect how strongly or weakly, respectively, the individual parameters change (in the most cases decrease) with degradation. Despite the simple definition the main problem is to fix the thresholds, where the resistance ends and the sensitivity starts. Consequently, a comparison between soil properties is necessary.

We suggest using SOC content as a universal reference soil property ${ }^{[13]}$ for four reasons. (1) SOC is sensitive to agricultural use and most forms of soil degradation. (2) SOC content defines various other physical, chemical and biological properties. (3) It is closely correlated to many properties (nutrient stocks and availability, bulk density and aggregation, CEC and sorption capacity, e.g., heavy metals and pesticides, and porosity). SOC is therefore effectively a master soil property ${ }^{[9]}$. (4) SOC has been used in most previous SQI ${ }^{[1]}$. Accordingly, all parameters for which the sensitivity and resistance should be evaluated will be presented orthogonal to the changes (decreases in the most degradation cases) of SOC (Fig. 4). 


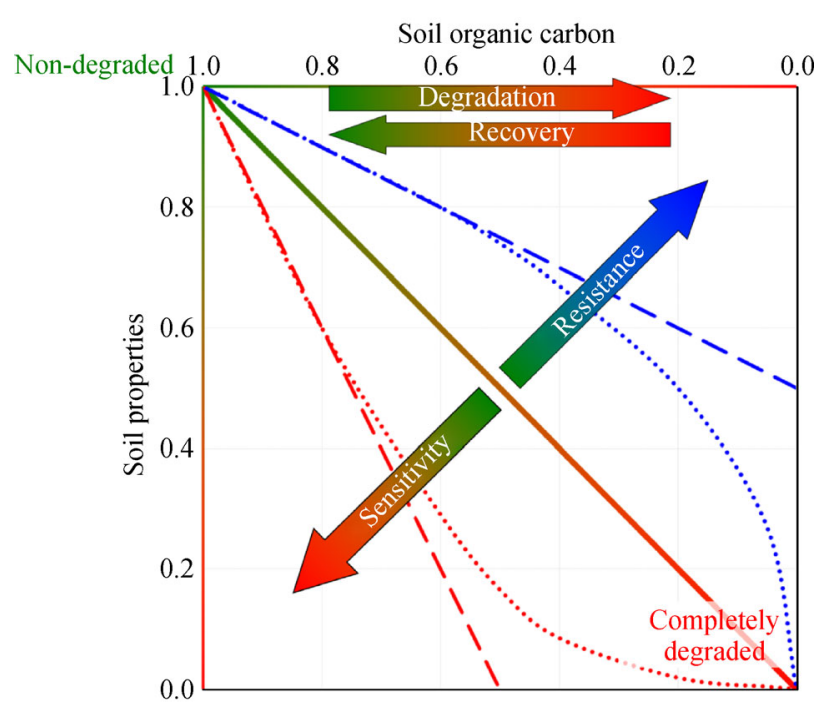

Fig. 4 Concept and evaluation principle of sensitivity and resistance of soil parameters to degradation or land-use change based on the comparison with soil organic carbon (SOC) content changes. Adapted from Guillaume et al. ${ }^{[13]}$, with permission from Elsevier. Soil properties can decrease proportionally with the decrease of SOC content (identity line, 1:1), or be resistant (above the identity line, $1: 1$ ) or sensitive (below the identity line, $1: 1$ ) compared to the SOC. The sensitivity or resistance of a property is either independent of SOC loss intensity (dashed lines) or dependent on SOC loss intensity (dotted lines). All properties are standardized to non-degraded reference soil (1.0, see Eq. (1)) and can decrease to 0 . For soil properties (Y scale), any soil parameters (e.g., involved in the calculation of SQI) can be used. Usually, (micro)biological properties are more sensitive and physical properties are more resistant to soil degradation.

If the property decreases proportionally to the SOC decrease (identity line on Fig. 4), it has the same sensitivity and resistance as the SOC. If the changes are faster than that of SOC (dashed line below the identity line), this property is sensitive, or if slower (above the identity line), the property is resistant to the degradation compared to SOC.

The dashed and dotted lines (Fig. 4) present specifics of the decrease in various soil parameters. Some degradation resistant parameters can remain in soil even if the SOC is substantially decreased or completely removed. These parameters include total phosphorus content, CEC, clay content and water retention, i.e., mainly the parameters related to parent materials. The other degradation sensitive parameters will reach levels close to zero before SOC is completely lost, e.g., microbial biomass, dissolved organic matter, activities of most enzymes and basal respiration, i.e., mainly the (micro)biological parameters ${ }^{[13,18,19]}$. Both groups of parameters, remaining in soil after SOC is completely lost or reaching zero before SOC is completely lost, are presented with dashed lines (Fig. 4). In contrast, some other parameters, despite sensitivity or resistance, are also reaching zero or are declining to the parent rock level only if SOC is lost completely (dotted lines on the Fig. 4). These parameters include total $\mathrm{N}$ content, macroaggregates, organo-mineral fractions and others. However, the sensitivity or resistance of soil properties does not reflect their resilience. Therefore, as SOC is responsible for a broad range of soil properties and functions, the comparison of any parameter with the SOC decrease by degradation, reflects its sensitivity or resistance.

Experimental applications of this approach using the same data as in Fig. $2^{[10]}$ give excellent separation of the sensitive and resistant soil properties for the recovery chronosequence of Phaeozems, but not very well for the Chernozems (Fig. 5). Basal respiration and free light fraction of organic matter $\left(\rho<1.6 \mathrm{~g} \cdot \mathrm{cm}^{-3}\right)$ were sensitive by Phaeozem recovery. Bulk density, water holding capacity (WHC), microbial biomass $\mathrm{C}$ (Cmic) and total $\mathrm{N}$ (TN) were resistant to recovery (Fig. 5(a)). Water holding capacity and bulk density remained resistant parameters by the Chernozem recovery. The response of other parameters by Chernozem recovery was not clear (Fig. 5(b)).

\section{Conclusions}

Evaluation of soil health, i.e., its multifunctionality and ability to provide a broad range of ecosystem services, remains challenging as no universal SQI have been developed and likely cannot be generated. The absence of universal SQI is connected with the fact that each quality indicator is specific and is aimed at a limited number of certain soil functions. To unify the application of various SQI, we suggest a universal approach based on the area of the radar plots considering any number of parameters contributing to the SQI. The area covered by parameters in degraded soil will be related to the nondegraded (natural) reference soil and the overall decrease in all properties or functions will be calculated. In the absence of a reference, various soils with increasing degradation levels can be compared using the same approach.

The sensitivity and resistance of any soil parameter can easily be evaluated based on the comparison of its decrease relative to the decrease of SOC content. Considering previous studies, we conclude that the most physical soil parameters are usually more resistant, and most biological parameters are more sensitive to degradation compared to SOC content. This sensitivity-resistance approach can be well used also to compare the relative sensitivity of various soils to degradation. Both approaches, the SQI-area and the sensitivity-resistance can be well used not only for basic and applied research questions, but also are attractive to decision-makers as they provide simple and unified 


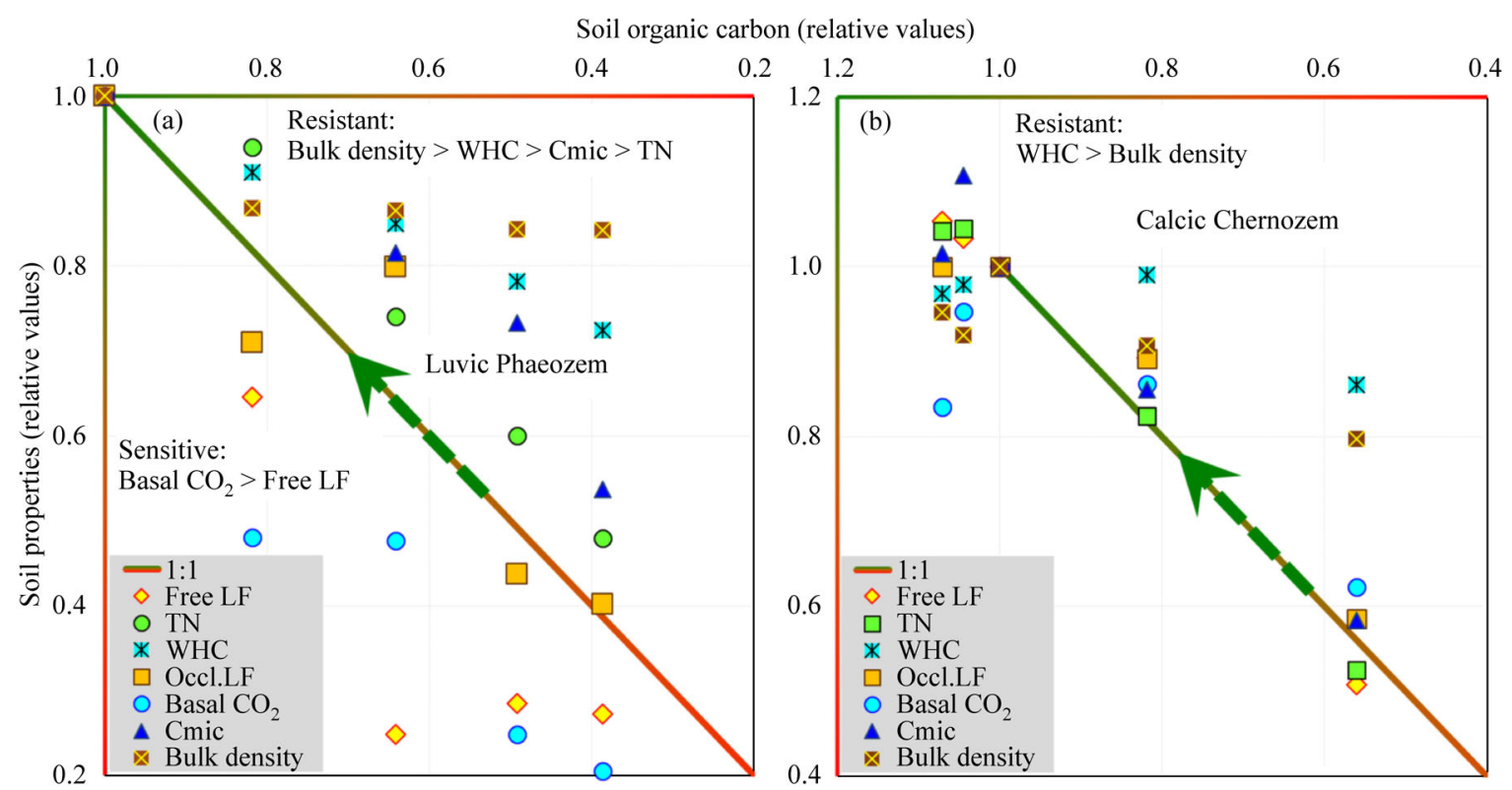

Fig. 5 Experimental evaluation of sensitivity and resistance of soil parameters to degradation or land-use change based on the comparison with changes of soil organic carbon (SOC) content during recovery of agricultural soils after abandonment and natural succession under deciduous forest: Luvic Phaeozem (a); and under dry steppe: Calcic Chernozem (b) (data from Ovsepyan et al. ${ }^{[10]}$ ). The green arrows reflect the direction of development of SOC and all soil parameters during recovery. For detailed explanations see Fig. 4. The sensitive and resistant parameters are presented for each soil.

options to evaluate the land-use practices and measures against soil degradation, and thus, soil health.

Acknowledgements We are most grateful for the state assignment AAAAA18-118013190177-9 and the Russian Foundation for Basic Research (18-04-00773 and 19-29-05260).

Compliance with ethics guidelines Yakov Kuzyakov, Anna Gunina, Kazem Zamanian, Jing Tian, Yu Luo, Xingliang Xu, Anna Yudina, Humberto Aponte, Hattan Alharbi, Lilit Ovsepyan, Irina Kurganova, Tida Ge, and Thomas Guillaume declare that they have no conflicts of interest or financial conflicts to disclose.

This article does not contain any studies with human or animal subjects performed by any of the authors.

\section{References}

1. Bünemann E K, Bongiorno G, Bai Z G, Creamer R E, De Deyn G, de Goede R, Fleskens L, Geissen V, Kuyper T W, Mader P, Pulleman M, Sukkel W, van Groenigen J W, Brussaard L. Soil quality - a critical review. Soil Biology \& Biochemistry, 2018, 120: $105-125$

2. Doran J W, Parkin T B. Defining and assessing soil quality. In: Doran J W, Coleman D C, Bezdicek D F, Stewart B A, eds. Defining Soil Quality for a Sustainable Environment. Madison: Soil Science Society of America, 1994, 3-21

3. Doran J W, Parkin T B. Quantitative indicators of soil quality: a minimum data set. In: Doran J W, Jones A J. eds. Methods for Assessing Soil Quality. Madison: Soil Science Society of America, 1996, 25-37
4. Gauthier S, Bernier P, Kuuluvainen T, Shvidenko A Z, Schepaschenko D G. Boreal forest health and global change. Science, 2015, 349(6250): 819-822

5. Reyer C P O, Brouwers N, Rammig A, Brook B W, Epila J, Grant R F, Holmgren M, Langerwisch F, Leuzinger S, Lucht W, Medlyn B, Pfeifer M, Steinkamp J, Vanderwel M C, Verbeeck H, Villela D M. Forest resilience and tipping points at different spatio-temporal scales: approaches and challenges. Journal of Ecology, 2015, 103 (1): 5-15

6. Milne E, Banwart S A, Noellemeyer E, Abson D J, Ballabio C, Bampa F, Bationo A, Batjes N H, Bernoux M, Bhattacharyya T, Black H, Buschiazzo D E, Cai Z C, Cerri C E, Cheng K, Compagnone C, Conant R, Coutinho H L C, de Brogniez D, de Carvalho Balieiro F, Duffy C, Feller C, Fidalgo E C C, da Silva C F, Funk R, Gaudig G, Gicheru P T, Goldhader M, Gottschalk P, Goulet F, Goverse T, Grathwohl P, Joosten H, Kamoni P T, Kihara J, Krawcaynski R, Scala Jr N L, Lemanceau P, Li L Q, Li Z C, Lugato E, Maron P A, Martius C, Melillo J, Montanarella L, Nikolaidis N, Nziguheba G, Pan G X, Pascual U, Paustian K, Pineiro G, Powlson D, Quiroga A, Richter D, Sigwalt A, Six J, Smith J, Smith P, Stacking M, Tanneberger F, Termansen M, van Noordwijk M, van Wesemael B, Vargas R, Victoria R L, Waswa B, Werner D, Wichmann S, Wichtmann W, Zhang X H, Zhao Y C, Zheng J W, Zheng J F. Soil carbon, multiple benefits. Environmental Development, 2015, 13: 33-38

7. Keesstra Saskia D, Bouma J, Wallinga J, Tittonell P, Smith $\mathrm{P}$, Cerdà A, Montanarella L, Quinton J N, Pachepsky Y, van der Putten W H, Bardgett R D, Moolenaar S, Mol G, Jansen B, Fresco L O. The significance of soils and soil science towards realization of the 
United Nations Sustainable Development Goals. Soil, 2016, 2(2): 111-128

8. Schwilch G, Bernet L, Fleskens L, Giannakis E, Leventon J, Marañón T, Mills J, Short C, Stolte J, van Delden H, Verzandvoort S. Operationalizing ecosystem services for the mitigation of soil threats: a proposed framework. Ecological Indicators, 2016, 67: 586-597

9. Kuzyakov Y, Zamanian K. Reviews and syntheses: Agropedogenesis-Humankind as the sixth soil-forming factor and attractors of agricultural soil degradation. Biogeosciences, 2019, 16 (24): 4783-4803

10. Ovsepyan L, Kurganova I, Lopes de Gerenyu V, Kuzyakov Y. Recovery of organic matter and microbial biomass after abandonment of degraded agricultural soils: the influence of climate. Land Degradation \& Development, 2019, 30(15): 1861-1874

11. Andrews S S, Karlen D L, Mitchell J P. A comparison of soil quality indexing methods for vegetable production systems in Northern California. Agriculture, Ecosystems \& Environment, 2002, 90(1): 25-45

12. Andrews S S, Flora C B, Mitchell J P, Karlen D L. Growers' perceptions and acceptance of soil quality indices. Geoderma, 2003, 114(3-4): 187-213

13. Guillaume T, Maranguit D, Murtilaksono K, Kuzyakov Y. Sensitivity and resistance of soil fertility indicators to land-use changes: new concept and examples from conversion of Indonesian rainforest to plantations. Ecological Indicators, 2016, 67: 49-57

14. Ovsepyan L A, Kurganova I N, Lopes de Gerenyu V O, Rusakov A V, Kuzyakov Y V. Changes in the fractional composition of organic matter in the soils of the forest-steppe zone during their postagrogenic evolution. Eurasian Soil Science, 2020, 53(1): 50-61

15. Glover J D, Reganold J P, Andrews P K. Systematic method for rating soil quality of conventional, organic, and integrated apple orchards in Washington State. Agriculture, Ecosystems \& Environment, 2000, 80(1-2): 29-45

16. Andrews S S, Carroll C R. Designing a soil quality assessment tool for sustainable agroecosystem management. Ecological Applications, 2001, 11(6): 1573-1585

17. de Bruyn L A L, Abbey J A. Characterisation of farmers' soil sense and the implications for on-farm monitoring of soil health. Australian Journal of Experimental Agriculture, 2003, 43(3): 285-305

18. Ros M, Hernandez M T, Garcia C. Soil microbial activity after restoration of a semiarid soil by organic amendments. Soil Biology \& Biochemistry, 2003, 35(3): 463-469

19. Trasar Cepeda C, Leiros C, Gil Sotres F, Seoane S. Towards a biochemical quality index for soils: an expression relating several biological and biochemical properties. Biology and Fertility of Soils, 1997, 26(2): 100-106 